\title{
Edificios de Oficinas bajo lineamientos de Energía Cero en climas templados.
}

\section{Office Buildings under Zero Energy guidelines in temperate climates.}

Presentación: 06 y 07 de octubre del 2020

\section{Doctorando:}

\section{Micaela D'Amanzo}

Instituto Ambiente, Hábitat y Energía (INAHE), Centro Científico Tecnológico (CCT) - Consejo Nacional de Investigaciones Científicas y Técnicas (CONICET) Mendoza - Argentina

mdamanzo@mendoza-conicet.gob.ar

\section{Director/a:}

\section{Carolina Ganem Karlen}

Co-director/a:

\section{María Victoria Mercado}

\section{Resumen}

El consumo energético en edificios de oficinas es unos de los más altos comparado con otras tipologías edilicias. Los Edificios Energía Cero tienen el objetivo de disminuir el consumo energético y las emisiones de CO2 generadas por el sector. La calidad ambiental en el interior de los espacios de trabajo contribuye a mejorar el confort de los usuarios, la productividad y evitar las enfermedades relacionadas con el Síndrome del Edificio Enfermo (SBS). Las características climáticas de la ciudad de Mendoza poseen grandes ventajas para enfrentar las problemáticas mencionadas mediante el bioclimatismo. En países desarrollados existen políticas claras para avanzar hacia ciudades post carbono, considerando a los EEC para la renovación del sector edilicio. En el presente trabajo se comunica el estado del arte de la temática y se presentan las consideraciones a tener en cuenta en la investigación, sumado a la necesidad de determinar una metodología soporte para la transformación de los edificios de oficinas del Área Metropolitana de Mendoza.

Palabras clave: Edificios Energía Cero, Eficiencia Energética, Sustentabilidad Edilicia.

\begin{abstract}
Energy consumption in office buildings is one of the highest compared to other building typologies. Zero Energy Buildings aims to reduce energy consumption and $\mathrm{CO} 2$ emissions generated by the building sector. Environmental quality inside workspaces contributes to improving user comfort, productivity, and avoiding illnesses related to Sick Building Syndrome (SBS). The climatic characteristics of the city of Mendoza have great advantages to face the mentioned issues through bioclimatism. In developed countries there are clear policies towards post- carbon cities, considering ZEBs for the renovation of the building sector. In this work the state of the art of the subject and the considerations to be taken into account in the research, together with the need of determine a support methodology for the transformation of office buildings in the Metropolitan Area of Mendoza are presented.
\end{abstract}


Keywords: Zero Energy Buildings, Energy Efficiency, Building Sustainability

\section{Introducción}

A partir de la última década, el ambiente construido ha sido responsable de gran parte del deterioro ambiental que genera el cambio climático, siendo el consumidor del $40 \%$ de la energía primaria mundial (Garde et al., 2014) y productor del $33 \%$ de emisiones de Gas Efecto Invernadero (Rogelj et al., 2018). Siendo que las emisiones globales de GEI de los edificios continuarán aumentando anualmente un promedio de 1.5\%(PérezLombard, Ortiz, \& Pout, 2008).

La aceptación global de los edificios verdes y el creciente desarrollo de medidas de eficiencia energética para edificios comerciales y públicos, provee un camino importante para incrementar seguridad energética, disminuir la demanda energética edilicia, mitigar emisiones de Gas de Efecto Invernadero y reducir el consumo para nuevas posibilidades de producción y distribución de energía (IEA, 2015).

El consumo energético en edificios de oficinas es unos de los más altos comparado con otras tipologías edilicias. El consumo anual varía entre 100 y $1000 \mathrm{kWh} / \mathrm{m} 2$, dependiendo de la localización geográfica, uso y tipo de equipamiento, horarios de funcionamiento, tipo de envolvente , uso de sistemas de acondicionamiento climático (HVAC), tipo de iluminación, etc. (Santamouris \& Hestnes, 2002).

Diversos estudios han demostrado que la productividad en el trabajo soporta una relación estrecha con el ambiente (Clements Croome et al. 2000) y con las enfermedades relacionadas con los edificios (Kalbasi, Ruhani, \& Rostami, 2019).

El diseño apropiado del espacio arquitectónico de oficinas, considerando estrategias de arquitectura bioclimática y sustentable, puede incrementar la productividad y el rendimiento, mejorar la fluidez comunicativa y optimizar la motivación de los trabajadores a la hora de desempeñar sus funciones (Hernández, 2013).

Las condiciones climáticas de la ciudad de Mendoza, zona de clima templado, presentan alta potencialidad de climatizar naturalmente los edificios, debido a los niveles de radiación solar elevados, la poca nubosidad, la amplitud térmica, aire frío nocturno en verano y los periodos estacionarios que permiten al diseñador optar por estrategias claramente diferenciadas a la hora de calefaccionar y enfriar. La planificación integral, el monitoreo y el seguimiento en la vida útil del edificio permiten corregir y lograr mejores niveles de EE y Confort (Kuchen, Plesser, \& Fisch, 2012).

Se propone definir la tecnología de Energía Cero Neta, sus posibilidades energéticas- ambientales aplicado a edificios de oficinas en el área Metropolitana de Mendoza, con el fin de promover una mejora sustancial en la relación entre el hombre, su espacio de trabajo y su entorno.

\section{Desarrollo}

Los Edificios Energía Cero (Zero Energy Buildings- ZEBs), también llamados edificios de Energía positiva, Edificios de baja energía o Ecológicos, son edificios que promueven una mirada integral de la arquitectura sustentable y un cambio profundo en la manera de construir (Berardi, 2018).

Desde los inicios, Torcellini, Pless, \& Deru, (2006) hasta la actualidad con (Attia, 2018), entre otros afirman que su objetivo es disminuir el consumo energético, a través de medidas pasivas y otras tecnologías con el fin de reducir, reutilizar y producir energía. Con ello alcanzar un balance energético neutro entre demanda y consumo sobre una base anual y minimizar el impacto ambiental edilicio. 
La Directiva 2010/31/UE del Parlamento Europeo y del Consejo. Los define como "casi nula o muy pequeña cantidad de energía requerida debe ser cubierta por una significante cantidad de energía de fuentes renovables, producidas en el sitio o cercano al mismo" (Comisión Europea, 2010).

Torcellini, Pless \& Deru (2006), desarrollan definiciones sobre el intercambio edificio- red energética para su abastecimiento. La figura 3 muestra la clasificación realizada por los autores según las condiciones de provisión energética de los edificios, energías renovables en el sitio o fuera del mismo.

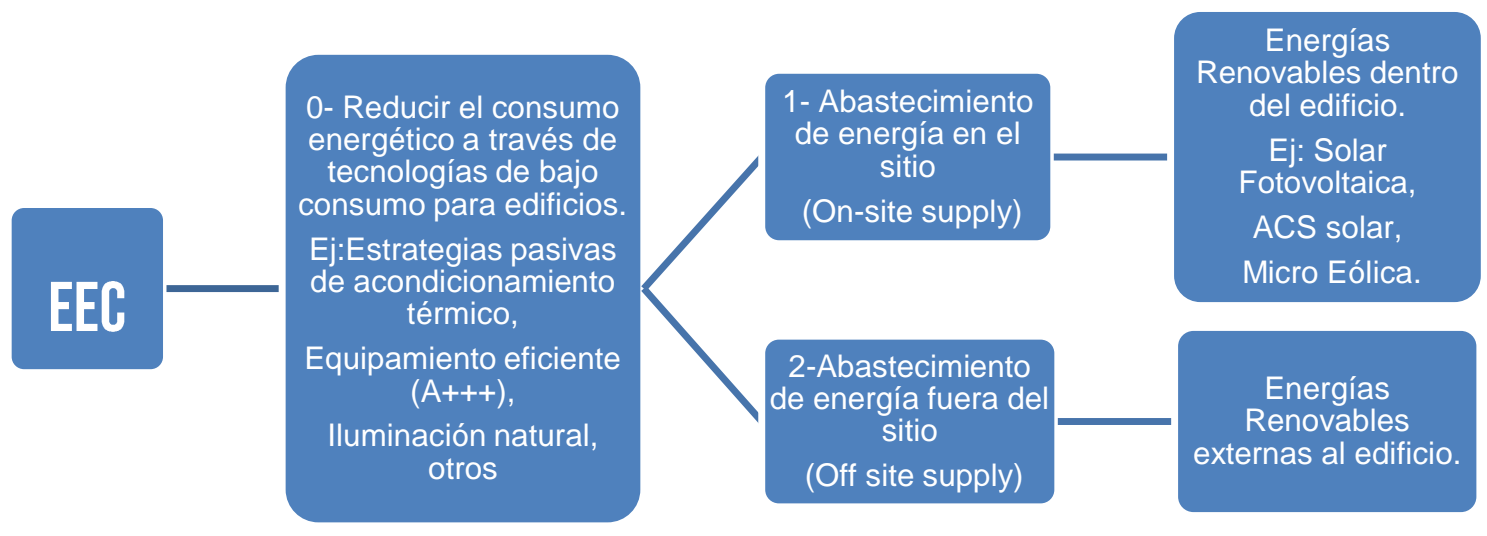

Figura 3: Interpretación de la clasificación de Torcellini et.al, (2006).

En algunos casos las opciones de generación de energía en el sitio son limitadas, por lo tanto resulta importante contemplar las características de la envolvente edilicia, las condiciones internas del edificio y los sistemas de climatización y servicios según las tecnologías disponibles en el lugar de emplazamiento del edificio (Li, Yang, \& Lam, 2013).

Los EEC se destacan mediante dos condiciones: el logro de un balance energético neutro entre la generación, y la demanda y la disminución de la huella de carbono del edificio durante todo su ciclo de vida (Chastas, Theodosiou, \& Bikas, 2016; Ibn-mohammed, 2017). Con el objetivo de minimizar la demanda energética, maximizar la producción de energía y los niveles de confort térmico(Giouri, Tenpierik, \& Turrin, 2020).

El mayor potencial en ahorro energético se encuentra en reducir la demanda de calefacción y refrigeración (Garde et al., 2014). Un edificio en climas templados con la aislación apropiada podría reducir la demanda de calefacción de un 20 a un 50\% (Taleghani, Tenpierik, Kurvers, \& Dobbelsteen, 2013).

\section{- El confort adaptativo y la habitabilidad en el espacio laboral.}

Según Nicol \& Humphreys (2002), la sustentabilidad debe ser considerada en la determinación del clima interior de los edificios prefiriendo soluciones disponibles de baja energía, ya que los estándares de confort pueden afectar el consumo energético.

El entendimiento del ambiente interior es un pre requisito para mejorar las condiciones actuales y establecer un ambiente confortable y sano para sus ocupantes, en cuanto a la eficiencia energética y el costo efectivo (Antoniadou \& Papadopoulos, 2017). Una definición genérica del término adaptación podría ser una disminución gradual de la respuesta del organismo a una estimulación repetida del ambiente (Muñoz, 2012). 
La tipología de oficinas ha modificado su arquitectura en relación a la funcionalidad a través del tiempo. Inicialmente la construcción se basaba en plantas sectorizadas según actividades, en la actualidad se fomenta la generación de espacios de planta libre donde se fomenta la vinculación de áreas (Hernández Chávez, 2002). Si bien se busca el rendimiento laboral de sus ocupantes, la literatura nos indica que esto no se logrará sin confort. Hoy prevalecen intereses como el trabajo creativo individual y la privacidad (Hernández, 2013).

Para evaluar el comportamiento energético para un edificio de oficinas energía cero bajo condiciones climáticas específicas en Bajenaru, Damian, \& Frunzulica, (2016), por ejemplo, mediante simulación computacional integran un sistema de ventilación mixto al edificio, donde asegura el confort térmico a sus ocupantes de acuerdo con la norma ASHRAE 55/2010, con un consumo racional de energía y un impacto ambiental mínimo.

Clements Croome, (2006) concluyen que el diseño arquitectónico puede incrementar la productividad o el rendimiento, mejorar la fluidez comunicativa y optimizar la motivación de los trabajadores a la hora de desempeñar sus funciones.

\section{Conclusiones}

Los países desarrollados presentan objetivos claros hacia las ciudades post carbono, considerando la transformación del sector terciario, que incluye a los edificios de oficinas. Se observa la necesidad de determinar una metodología soporte en el tema a nivel local y de referencia para futuros ajustes a nivel nacional en edificios de oficinas.

En la investigación doctoral se trabaja en una hipótesis de causalidad multivariada, de causa y efecto entre la energía cero, el consumo energético, el ambiente interno- externo, confort, producción del trabajo y valor económico. Donde se espera que el desarrollo de arquitectura de Energía Cero, energéticamente sustentable y económicamente factible, producirá una disminución en los consumos energéticos y el impacto ambiental en edificios de oficinas, mejorando ambiente interno y externo, favoreciendo las condiciones de confort de sus ocupantes, incrementando la eficiencia en la producción del trabajo y potenciando el valor económico del edificio en el mercado.

Como contribución se espera determinar un valor de consumo casi nulo de energía alcanzable para la provincia, que pueda ser utilizado como base de certificación de edificios Energía Cero Neta y fomentar buenas practicas para la construcción en el AMM para minimizar las emisiones de CO2 y el consumo energético en áreas urbanas.

\section{Referencias}

Antoniadou, P., \& Papadopoulos, A. M. (2017). Occupants ' thermal comfort : State of the art and the prospects of personalized assessment in office buildings. Energy \& Buildings, 153, 136-149. https://doi.org/10.1016/j.enbuild.2017.08.001

Attia, S. (2018). Net Zero Energy Buildings (NZEB) : concepts, frameworks and roadmap for project analysis and implementation.

Bajenaru, N., Damian, A., \& Frunzulica, R. (2016). Evaluation of the Energy Performance for a nZEB Office Building under Specific Climatic Conditions. Energy Procedia, 85(November 2015), 26-34. https://doi.org/10.1016/j.egypro.2015.12.271

Berardi, U. (2018). ZEB and nZEB (definitions, design methodologies, good practices, and case studies) Optimization of the Effective Performance of New Foam-based Building Insulation View project ZEB and nZEB (definitions, design methodologies, good practices, and case studi. In Preprint in Handbook of 
Energy Efficiency in Buildings (2018 Elsev). https://doi.org/10.1016/B978-0-12-812817-6.00038-3

Chastas, P., Theodosiou, T., \& Bikas, D. (2016). Embodied energy in residential buildings-towards the nearly zero energy building: A literature review. Building and Environment, 105, 267-282.

https://doi.org/10.1016/j.buildenv.2016.05.040

Clements-Croome, D.J and LI, B. (2000), International Conference on Healthy Buildings 2000, August 6-10, Helsinki. Environmentalquality and the productive workplace.

Clements-Croome, Derek J. (2006). Creating the Productive Workplace, $2{ }^{a}$ ed. EEUU y Cánada: Taylor y Francis.

Comisión Europea. (2010). Directiva 2010/31/UE relativa a la eficiencia energética de los edificios. Boletin Oficial, L135, 13-35.

Garde, F., Lenoir, A., Scognamiglio, A., Aelenei, D., Waldren, D., Rostvik, H. N., ... Cory, S. (2014). Design of net zero energy buildings: Feedback from international projects. In Energy Procedia (Vol. 61). https://doi.org/10.1016/j.egypro.2014.11.1011

Giouri, E. D., Tenpierik, M., \& Turrin, M. (2020). Zero energy potential of a high-rise office building in a Mediterranean climate : Using multi-objective optimization to understand the impact of design decisions towards zero-energy high-rise buildings. Energy \& Buildings, 209, 109666.

https://doi.org/10.1016/j.enbuild.2019.109666

Hermelink, A., Schimschar, S., Boermans, T., Pagliano, L., Zangheri, P., Armani, R., ... Musall, E. (2013). Towards nearly zero- energy buildings Definition of common principles under the EPBD Final report. Http://Ec.Europa.Eu/Energy/Efficiency/Buildings/Doc/Nzeb_full_report.Pdf.

Hernández Chávez, V. (2002). La habitabilidad energética en edificios de oficinas. Tesis Doctoral-Universidad Politecnica de Cataluya. Retrieved from http://search.ebscohost.com/login.aspx?direct=true\&site=edslive\&db=edstdx\&AN=tdx.10803.6107 OP - TDX (Tesis Doctorals en Xarxa)

Ibn-mohammed, T. (2017). Application of mixed-mode research paradigms to the building sector: A review and case study towards decarbonising the built and natural environment. Sustainable Cities and Society, 35(September), 692-714. https://doi.org/10.1016/j.scs.2017.09.027

IEA. (2015). SOLAR HEATING AND COOLING PROGRAMME. Task 40 (EBC Annex 52) Towards Net Zero Energy Solar Buildings. Retrieved from http://task40.iea-shc.org/Data/Sites/1/publications/IEA-SHCNZEB-Position-Paper.pdf

Kalbasi, R., Ruhani, B., \& Rostami, S. (2019). Energetic analysis of an air handling unit combined with enthalpy air - to - air heat exchanger. Journal of Thermal Analysis and Calorimetry. https://doi.org/10.1007/s10973-019-09158-9

Kuchen, E., Plesser, S., \& Fisch, M. N. (2012). Eficiencia Energética y Confort Edificios de Oficina : el Caso Alemán en Eficiencia Energética y Confort en Edificios de Oficina : el Caso Alemán Energy Efficiency and Comfort in Office Buildings : the German Case. Hábitat Sustentable, 2, 34-44.

Li, D. H. W. W., Yang, L., \& Lam, J. C. (2013). Zero energy buildings and sustainable development implications e A review. Energy, 54, 1-10. https://doi.org/10.1016/J.ENERGY.2013.01.070

Muñoz, A. G. (2012). El confort térmico adaptativo Aplicación en la edificación en España.

Nicol, J. F., \& Humphreys, M. A. (2002). Adaptive thermal comfort and sustainable thermal standards for buildings, 34, 563-572.

Pérez-Lombard, L., Ortiz, J., \& Pout, C. (2008). A review on buildings energy consumption information. Energy 
and Buildings, 40(3), 394-398. https://doi.org/10.1016/j.enbuild.2007.03.007

Rogelj, J., Shindell, D., Jiang, K., Fifita, S., Forster, P., Ginzburg, V., ... Vilariño, M. V. (2018). Mitigation Pathways Compatible with $1.5^{\circ} \mathrm{C}$ in the Context of Sustainable Development. Global Warming of $1.5^{\circ} \mathrm{C}$. An IPCC Special Report on the Impacts of Global Warming of $1.5^{\circ} \mathrm{C}$ above Pre-Industrial Levels and Related Global Greenhouse Gas Emission Pathways, in the Context of Strengthening the Global Response to the Threat of Climate Change,.

Santamouris, M., \& Hestnes, A. G. (2002). Office-passive retrofitting of o ce buildings to improve their energy performance and indoor working conditions. Building and Environment, 37(6), 555-556. https://doi.org/10.1016/S0360-1323(02)00038-0

Taleghani, M., Tenpierik, M., Kurvers, S., \& Dobbelsteen, A. Van Den. (2013). A review into thermal comfort in buildings. Renewable and Sustainable Energy Reviews, 26, 201-215.

https://doi.org/10.1016/j.rser.2013.05.050

Torcellini, P., Pless, S., \& Deru, M. (2006). Zero Energy Buildings : A Critical Look at the Definition. 